\title{
A Design of Power Supply Circuit Module of Fuse General Tester
}

\author{
Hu Longtao, Fan Tiansuo, Zuo Dongguang, Yuan Ding, Li fengchen \\ Xi'an Research Institute of High Technology, Xi'an 710025, China \\ 523098802@qq.com
}

Keywords: fuse; Circuit; Stable voltage; Circuit Design; chip circuit design

\begin{abstract}
In order to solve the problem that conventional single IPC tester exists a universal machine a difficult test, a large space and other issues, the researchers designed a new fuse covers a variety of common tester, according to several types of fuses and general target test tester itself different actual voltage required to design universal power supply module required circuit tester, using URB2405MT-3WR3, L78M09ABDT, DCP010505B, ADUM1200 and many other models NCP117ST18T3 chip circuit design, to ensure sustained and stable to provide voltage tester and test Fuse eliminate glitches affected. Experimental data show that: the power circuit design meets the requirements, to provide for the tester and test fuse all required sustained high and low voltage, the power supply circuit to provide a reference for future Fuse Test and simulation.
\end{abstract}

\section{Introduction}

In order to solve the conventional single IPC tester exists a universal machine a difficult test, a large space and other issues, an urgent need to design a study to cover a variety of Fuse universal tester, and capable of power, control display, real-time identification, telemetry Jaguar and simulation, zero detect people and other tasks demand modular design in order to meet easy maintenance, intelligent detection, general good requirements. Since several types of fuses and general target test tester tester itself required voltage is different, it is necessary to design a set of power supply circuits for the target institution to provide power fuse tester universal power supply module according to the needs of each target organization nowhere, adopt URB2405MT-3WR3, L78M09ABDT, DCP010505B, ADUM1200 and NCP117ST18T3 circuit chips and other related design models.

Power module circuit design used to have the following salient features:

(1) to the tester, the sustained and stable supply voltage fuse, to maintain the accuracy within $3 \%$;

(2) having an input voltage protection, output short circuit, overcurrent protection;

(3) resistance to harsh environment, strong anti-interference;

(4) affordable, suitable for bulk purchases.

\section{Organization of the Text}

\section{Tester power module requirements}

Fuse tester consists of three parts and control unit, and the power supply unit into the machine interaction and other components composition, theory composition shown in Figure 1.

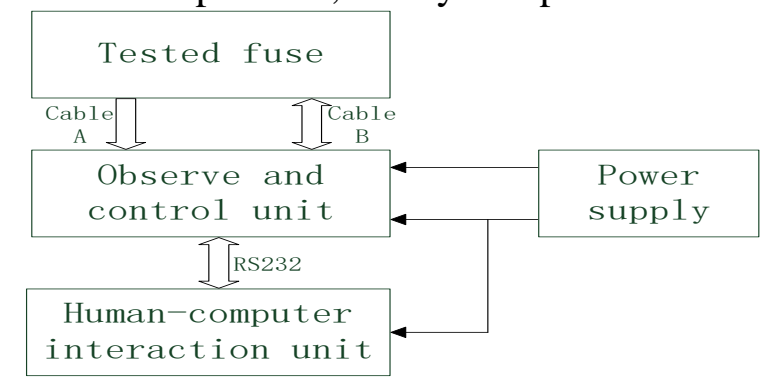

Fig.1 Fuse tester block diagram

The main power supply module selection isolated AC/DC, nominal output $28 \mathrm{~V}$, by adjusting the 
bias resistor can output $25 \mathrm{~V}$ or $32 \mathrm{~V}$, in addition to the main power supply fuses provide security solutions outside, but also to the individual modules DC/DC power supply to provide input . Wherein the power control function integrated security solutions in telemetry and analog modules.

Using AC/DC power supply as the main module by LM78M09 regulator will convert $28 \mathrm{~V} / 9 \mathrm{~V}$, then converted to $5 \mathrm{~V}$ supply telemetry module front-end, after isolation by $28 \mathrm{~V}$ conversion to $5 \mathrm{~V}$ DCDC-URB2405 four to a total of, respectively, to a total of four functions module.

A system configuration diagram of the power module shown in Figure 1, it can be seen, the power switch $220 \mathrm{~V} 50 \mathrm{~Hz}$ AC power is converted to $28 \mathrm{~V}$ DC voltage signal, $28 \mathrm{~V}$ DC voltage signal through the DC/DC switching power supply is divided into two respectively, through DC/DC isolation and voltage regulator modules to obtain multi-channel $5 \mathrm{~V}$ regulated DC voltage signal.

Wherein, DC / DC switching power supply 28V DC into 5V working power, as the newly developed miniaturized power switch module, which will be assembled using microelectronic technology integrated micro-electronic components and integrated circuits, an input voltage range more wide, the output voltage ripple is smaller, higher efficiency and other advantages. DC/DC isolation module transformer isolation so that the input and output voltage is not common ground, eliminating ground loops, so as to avoid distortion of the supply signal occurs during transmission. Regulator module is the role of voltage stability, providing stable $5 \mathrm{~V}$ voltage signal for the entire device.

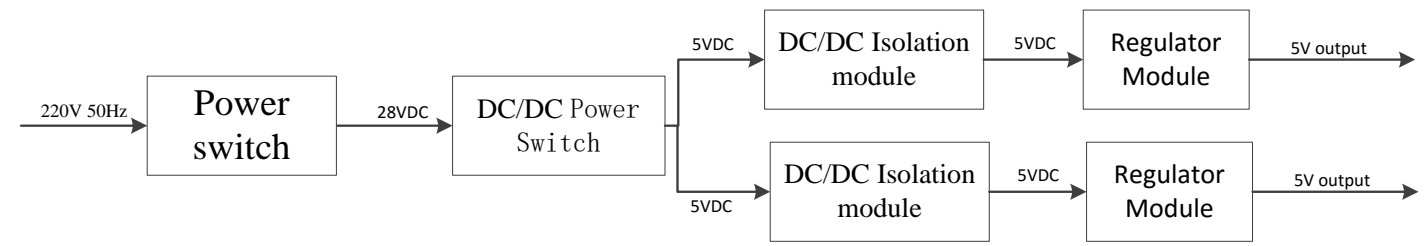

Fig.2 The overall operation of the power system block diagram

\section{Chip and circuit design}

Use L78M09ABDT, URB2405MT-3WR3, DCP010505B, ADUM1200 and NCP117ST18T3 circuit chips and other related design models, according to power requirements, circuit chip to have a strong supply capability under complex and harsh environments, when large current carrying capacity trigger higher.

\section{LM78M09 chip circuit design}

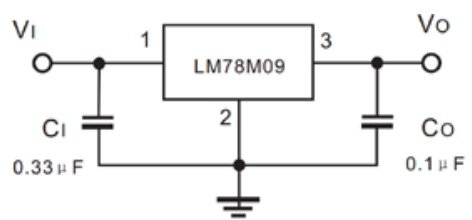

Fig.3 voltage stabilizer LM78M09

LM78M09 low dropout linear regulator is a new generation of integrated circuit voltage regulator, as shown in Fig3, three-terminal regulator it the biggest difference is that, as the micro-chip system, since the consumption of lower three correct voltage. Can be used to control current to the main channel, integrated on-chip mosfet with very low on-resistance line, Schottky diodes, and sampling resistor voltage divider resistors and other hardware circuit and over-current protection, over-temperature protection, precision reference, differential amplifier, delay and other functions. And a self-test each output state, delay safe power supply function. Also has very low self noise and high power supply rejection ratio, Tab1 LM78M09 low dropout linear regulator electrical characteristics. 
Tab.1 Electrical characteristics of L78M09

\begin{tabular}{clccc}
\hline parameter & \multicolumn{1}{c}{ Test Conditions } & Typical values & Found & unit \\
\hline Output voltage & & 9 & 9.35 & $\mathrm{~V}$ \\
Output voltage & $\mathrm{I}_{\mathrm{O}}=(5 \sim 350 \mathrm{~mA}), \mathrm{V}_{\mathrm{I}}=(10.5 \sim 24 \mathrm{~V})$ & 9 & 9.45 & $\mathrm{~V}$ \\
& $\mathrm{I}_{\mathrm{O}}=200 \mathrm{~mA}, \mathrm{~V}_{\mathrm{I}}=(11.5 \sim 25 \mathrm{~V})$ & & 180 & $\mathrm{mV}$ \\
Load Regulation & $\mathrm{I}_{\mathrm{O}}=200 \mathrm{~mA}$ & & 90 & $\mathrm{~mA}$ \\
Quiescent Current & & & 6.5 & $\mathrm{~mA}$ \\
Quiescent current changes & $\mathrm{I}_{\mathrm{O}}=(5 \sim 350 \mathrm{~mA})$ & & 0.8 & \\
Output voltage drift & $\mathrm{I}_{\mathrm{O}}=200 \mathrm{~mA}, \mathrm{~V}_{\mathrm{I}}=(11.5 \sim 25 \mathrm{~V})$ & -0.5 & & $\mathrm{mV} /{ }^{\circ} \mathrm{C}$ \\
Power Supply Rejection & $\mathrm{I}_{\mathrm{O}}=5 \mathrm{~mA}, \mathrm{~T}_{\mathrm{J}}=\left(0 \sim 125^{\circ} \mathrm{C}\right)$ & & $\mathrm{dB}$ \\
Output noise voltage & $\mathrm{V}_{\mathrm{I}}=(12.5 \sim 23 \mathrm{~V}), \mathrm{F}=120 \mathrm{~Hz}, \mathrm{I}_{\mathrm{O}}=300 \mathrm{~mA}$ & 58 & & $\mu \mathrm{V}$ \\
Short circuit current & $\mathrm{B}=10 \mathrm{~Hz} \sim 100 \mathrm{kHz}$ & $\left.\mathrm{V}_{\mathrm{I}}=35 \mathrm{~V}\right)$ & 250 & \\
\hline
\end{tabular}

(Referring to the test circuit, $\mathrm{TJ}=25^{\circ} \mathrm{C}, \mathrm{VI}=15 \mathrm{~V}, \mathrm{IO}=350 \mathrm{~mA}, \mathrm{Cl}=0.33 \mu \mathrm{F}, \mathrm{CO}=0.1 \mathrm{~F}$ )

LM78M09 by a low dropout linear regulator circuit used to build the universal tester main fuse module powered by $9 \mathrm{~V}$ power supply, voltage regulators on both sides of 28-volt converter mounted $47 \mu \mathrm{F}$ capacitor circuit design in Figure 4 as follows:

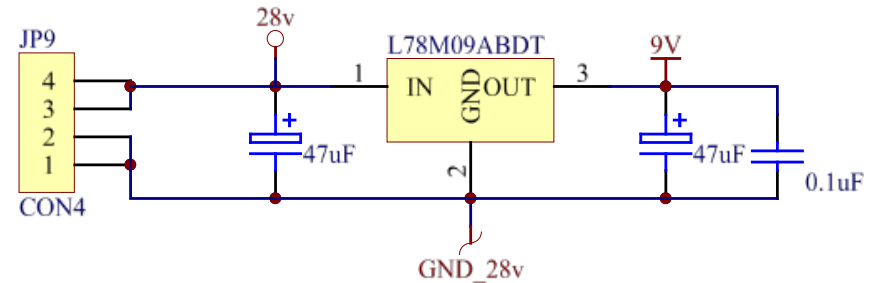

Fig.4 Design of Low Dropout Linear Regulator circuit based LM78M09

\section{URB2405MT-3WR3 chip circuit design}

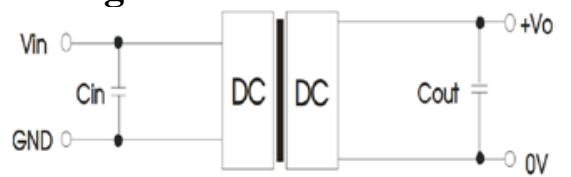

Fig.5 URB2405MT-3WR3 DC-DC Power Module

As shown in Fig5 URB2405MT-3WR3 DC-DC power module, the module voltage range (4:1), up to $84 \%$ efficiency, no-load power consumption as low as $0.12 \mathrm{~W}$, isolation voltage $1500 \mathrm{VDC}$, with input undervoltage protection, output short circuit, overcurrent protection, operating temperature $-40^{\circ} \mathrm{C} \sim+85^{\circ} \mathrm{C}$, small ripple noise, using the international standard pin mode, the output power of 3W, wide voltage input 9-36VDC, 18-75VDC, isolation voltage 1500VDC, widely used in industrial, electrical, instrumentation and communications fields, in general fuse tester output $5 \mathrm{~V}$ regulator can be used to design the four auxiliary module circuit. Tab2 shows the electrical characteristics URB2405MT-3WR3 power module.

Tab.2 Electrical characteristics of URB2405MT-3WR3

\begin{tabular}{|c|c|c|c|c|c|}
\hline \multicolumn{2}{|c|}{ Output voltage (V) } & \multicolumn{2}{|c|}{ Output } & \multirow{2}{*}{$\begin{array}{l}\text { Full load efficiency } \\
\text { (Min/Typ) }\end{array}$} & \multirow{2}{*}{$\begin{array}{c}\text { Maximum capacitive } \\
\text { load ( } \mu \mathrm{F})\end{array}$} \\
\hline $\begin{array}{l}\text { Nominal value } \\
\text { (Range of values) }\end{array}$ & Max & $\begin{array}{c}\text { Output } \\
\text { voltage (V) }\end{array}$ & $\begin{array}{l}\text { Output current } \\
\text { (Max/Min) }\end{array}$ & & \\
\hline $\begin{array}{c}24 \\
(9-36)\end{array}$ & 40 & 5 & $600 / 0$ & $78 / 80$ & 2200 \\
\hline
\end{tabular}

Fig 6 and Fig 7 URB2405MT-3WR3 input voltage and efficiency, the and Efficiency at full load ratio. 


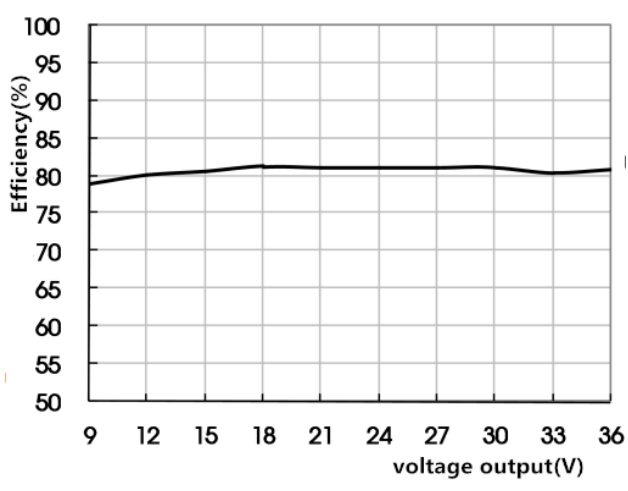

Fig.6 Efficiency Vs voltage output

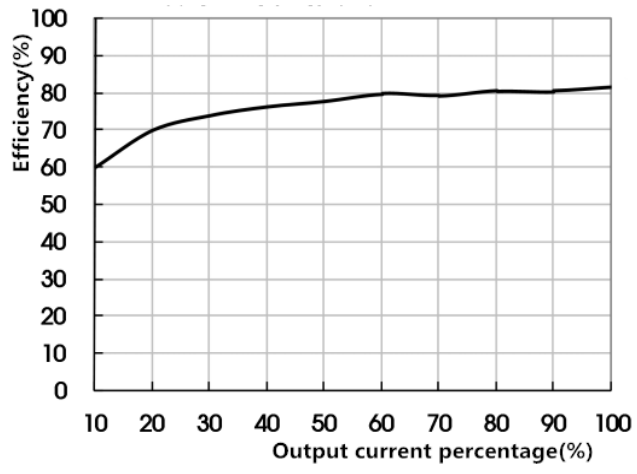

Fig7 Efficiency Vs Electric current output

Fig8 is equipped with URB2405MT-3WR3 design 5V regulator circuit, a total of four designs for four auxiliary module selection.

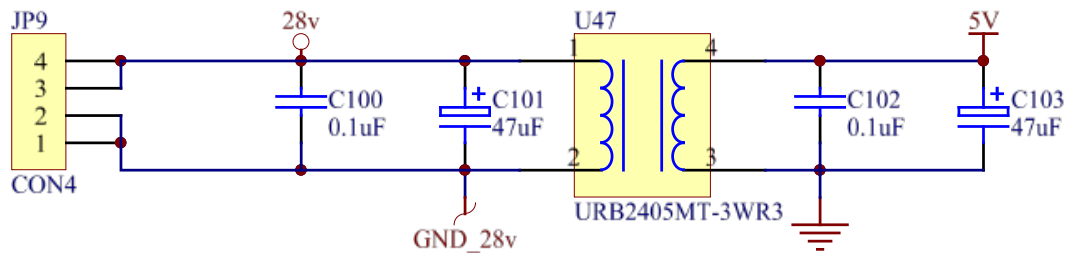

Fig8 Design of circuit based URB2405MT-3WR3

\section{DCP010505B chip circuit design}

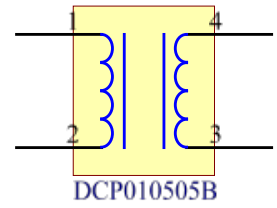

Fig.9 DCP010505B DC/DC isolation module

DCP010505B DC/DC isolation module is $1 \mathrm{~W}$ isolation, stabilization circuit to eliminate ground loop protection circuit Overload DC/DC converter module, requires minimal external components, including chip device protection, there is provided an additional as output disable and switching frequency synchronization and other functions. Excellent characteristics and compact size combination makes it widely used in electrical equipment, and can realize the signal path isolation, Table 3 DCP010505B DC/DC converter electrical characteristics, Fig10and Fig11 is DCP010505B DC/output voltage DC isolation module the relationship between efficiency and load.

Tab.3 Electrical characteristics of DCP010505B DC/DC isolation module

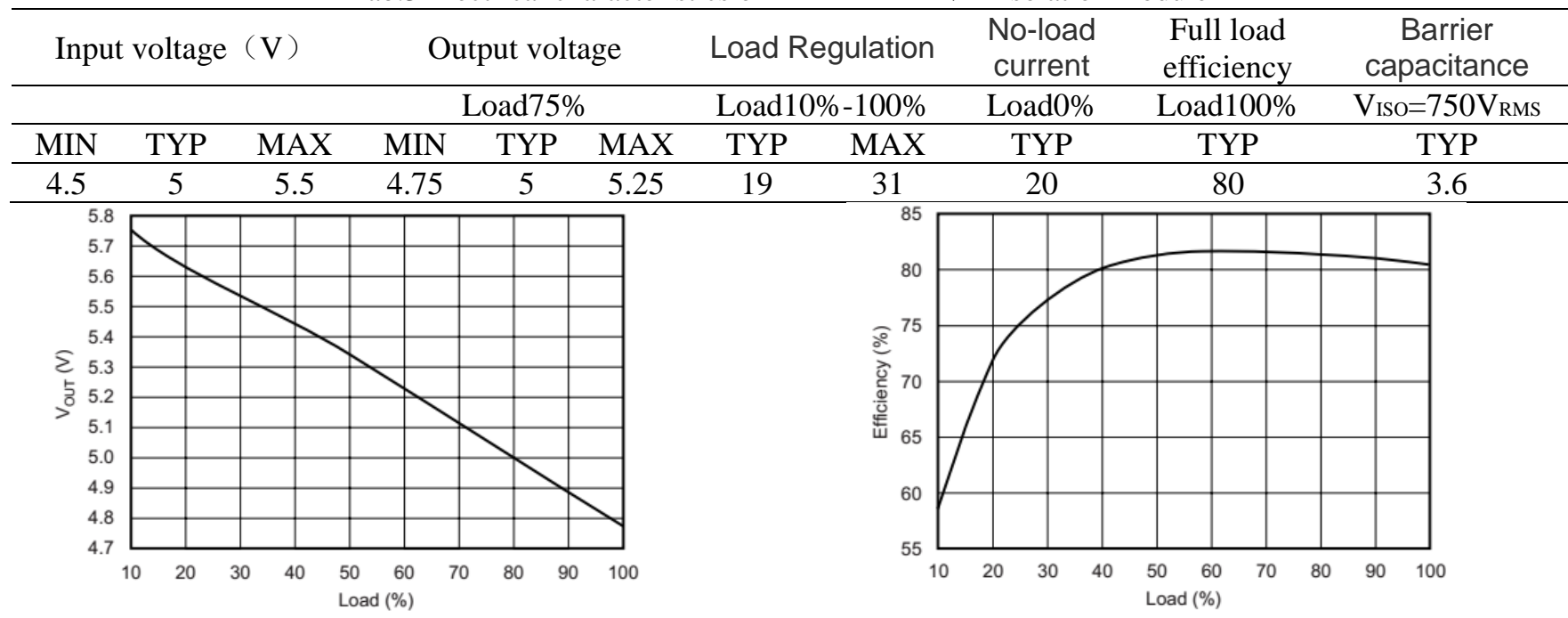

Fig.10 DCP010505B Vout Vs load

Fig.11 DCP010505B Efficiency vs load

Fig.11 is mounted DCP010505B DC/DC isolation module design 5V regulator circuit, mainly to provide $5 \mathrm{~V}$ voltage value is a second way power, stability and eliminates ground loop circuit, power 
supply protection circuit overload safety, ensuring stable synchronization with the switch.

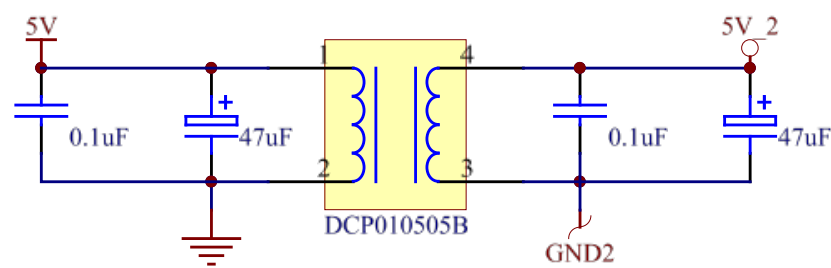

Fig.12 Design of circuit based DCP010505B DC/DC converter

\section{ADUM1200 chip circuit design}

13 is ADUM1200 isolator dual-channel isolator, for magnetic coupling for each channel comprising: an input buffer, an encoder (built refresh generator), an isolation transformer, a decoder (built gatekeeper Watchdog timer) and an output buffer. Because these are usually used in some magnetic coupling field bus, but in order to receive and send information to complete on the same piece of magnetic coupling, therefore it is necessary to have forward and reverse channels. Since the magnetic coupling using CMOS technology, in system level ESD (electrostatic discharge), surge voltage, fast transient or other overvoltage conditions, it is more susceptible to damage than an optocoupler or latch of ESD. Based on these magnetically coupled high-speed transfer rates and excellent isolation capabilities, they can protect the security and stability of the power supply system, such as RS-232 applications can use magnetic coupling.

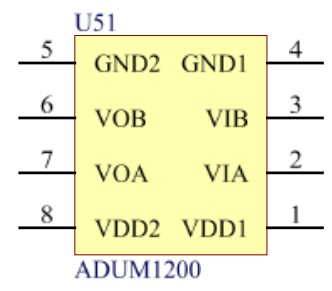

Fig.13 ADUM1200 Isolator

ADUM1200 isolator uses directional 2/0 channel dual-channel digital isolators based on ADI's iCoupler ${ }^{\circledR}$ technology. The isolation device will speed CMOS single-chip technology integrated with transformer, superior to alternatives such as optocoupler devices performance characteristics. The isolator without LED and photodiode, and thus there is no commonly associated with optocouplers design difficulties. Its relatively simple digital interfaces and stable performance characteristics, can eliminate the typical optocoupler uncertain current transfer ratios, nonlinear transfer functions, and temperature and lifetime effects. Product does not require external drivers and other discrete components. Device power consumption is only under the same conditions optocouplers $1 / 10$ to $1 / 6$.RoHS compliant, low-power, two-way communication, working temperature up to $125^{\circ} \mathrm{C}$, $3 \mathrm{~V} / 5 \mathrm{~V}$ level translation High temperature operation: $125{ }^{\circ} \mathrm{C}$, ADuM1200 isolators provide two independent isolation channels in a variety of channel configurations and data rate. $2.7 \mathrm{~V}$ to $5.5 \mathrm{~V}$ supply voltage, providing compatibility with lower voltage systems, and is capable of enabling voltage translation functionality across the isolation barrier. In addition, ADuM1200 has a low pulse width distortion (CR level of less than 3 ns) and tight channel matching (CR level of less than $3 \mathrm{~ns}$ ) characteristics. Unlike other optocoupler, ADuM1200 isolators have a patented refresh feature that ensures power and on / off dc correctness in the off condition when the input logic conversion is not exist. Application size critical multichannel isolation, SPI interface / data converter isolation, RS-232 / RS-422 / RS-485 transceiver isolation Digital field bus isolation and other fields.

In general fuse tester power module, equipped ADUM1200 isolators designed circuit provides $5 \mathrm{~V}$ main power supply for the state detection module, the design of the circuit shown in Fig 14. 


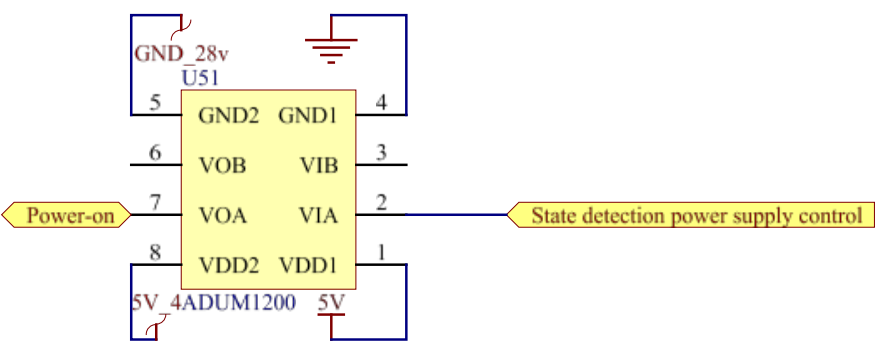

Fig.14 Design of circuit based ADUM1200 isolator

NCP117ST18T3 chip circuit design

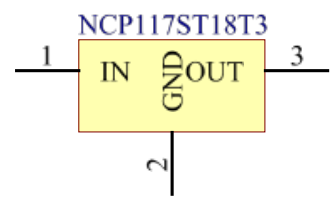

Fig.15 NCP117ST18T3 converter

Fig15 is a NCP117ST18T3 AC-DC converter, to ensure effective isolation output frequency range of $90 \mathrm{kHz} \sim 114 \mathrm{kHz}$, input voltage range of $11 \mathrm{~V} \sim 16 \mathrm{~V}$, operating temperature is $-25^{\circ} \mathrm{C} \sim$ $125^{\circ} \mathrm{C}$. 5V regulated to meet the design requirements, and stable performance, is mainly responsible LM78M09 low dropout linear regulator to convert the 9V secondary voltage conversion circuit design shown in Figure 16.

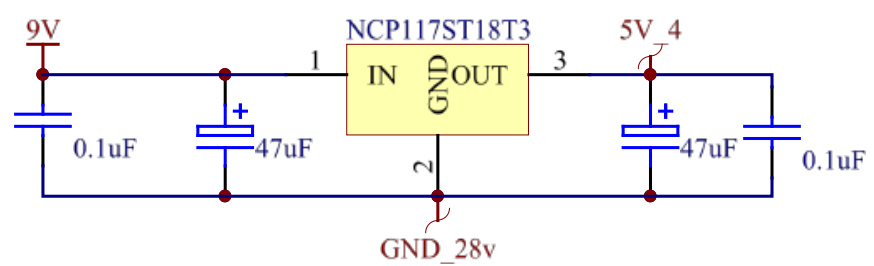

Fig.16 Design of circuit based NCP117ST18T3 converter

\section{Testing and certification}

By the above-described design of the electrical circuit and the output terminal voltage is measured, the analog output voltage test, the results shown in Table (wherein a plurality of different modules _ * represents the same power output circuits, respectively) 4.

Tab.4 Test table of power supply module circuit

\begin{tabular}{ccc}
\hline $\begin{array}{c}\text { Power output module } \\
\text { identification }\end{array}$ & Ideal value & test value \\
\hline 3.3V & $3.3 \mathrm{~V}$ & $3.30 \mathrm{~V}$ \\
3.3v_1 & $3.3 \mathrm{~V}$ & $3.28 \mathrm{~V}$ \\
$5 \mathrm{~V} \_2$ & $5 \mathrm{~V}$ & $5.02 \mathrm{~V}$ (on load) \\
& & $7.82 \mathrm{~V}$ (no load) \\
$5 \mathrm{~V} \_3$ & $5 \mathrm{~V}$ & $5.02 \mathrm{~V}$ \\
$5 \mathrm{~V}$ _4 & $5 \mathrm{~V}$ & $5.29 \mathrm{~V}$ \\
5V_5 & $5 \mathrm{~V}$ & $5.01 \mathrm{~V}$ \\
3.3V_6 & $3.3 \mathrm{~V}$ & $3.28 \mathrm{~V}$ \\
\hline
\end{tabular}

The test results show that the designed circuit satisfies a universal tester main module, attached to the module and the measured fuse provide sustained and stable voltage accuracy standard, maintained at less than $3 \%$, the function is completely resistant to harsh environment, pressure performance is good strong anti-interference, in line with standard power supply, you can use a universal power supply module fuse tester. 


\section{Summary}

This paper presents the structural analysis and circuit design of Fuse universal tester power supply module, and the output voltages of the respective circuits are measured, use several temperature span, more stable performance, the price is more economical, compact and powerful element, and a power supply circuit design. Measured data show that the designed circuit satisfies the functional requirements, to provide credible and reliable security and stability voltage tester and test fuses, instrument testing procedure to ensure no power failure occurs, the same type of tester for the future and associated circuitry design provides reference.

\section{References}

[1] Zhang Xionge. The Research and Design of Boundry Chekout Circuit Moudle[D]. Guangzhou: South China University of Technology, 2012.

[2] Wang Yixiao. Multiplexed Output DC/DC Power Module Design and Implementation [D]. XiAn: Xijing University, 2015.

[3] Si Ming. The Design of a PWM Control Circuit of The Switching Power Supply[D]. ShenYang: LiaoNing University, 2013.

[4] Zhang YaJun, Zhang Jianjun, Qi Hongxi ect. Automatic Detector of Muti-fuse Circuit Boards[J]. MINE WARFARE\&SHIP-DEFENCE, 2014, 22 (2): 30-33.

[5] Wang Shuilian. Development of special purpose fuse testing system [D]. Harbin Institute Of Technology, 2013.

[6] Zhanwen XI, Weirong NIE, Qilei LI. A MEMS Interrupter Mechanism for Fuse Safety \& Arming Device [J]. International Conference on Mechanical Engineering and Mechanics, 2009,3:51-56. 